\title{
Preference of chicks in the original stimulus situation of imprinting'
}

\author{
Tom L. Smith and Merle E. Meyer \\ WHITMAN COLLEGE
}

\begin{abstract}
Abstraet
Three factors of sound, color, and form were tested in a modified Gray-Pattie choice apparatus for the effects upon 64 chicks in regard to preference behavior. Sound and color were found to be significant with color being more important than sound. Form and all interactions were nonsignificant, though the effects of the stimuli were additive. The results were seen in light of a hypothesis relating arousal and imprinting.

\section{Problem}

That a wide variety of objects may elicit the following response of the imprinting process is demonstrated by various reports (i. e., Hinde, Thorpe, \& Vince, 1956; Hess, 1962; Ramsay, 1951). Color as a dimension of the stimulus has received a great deal of attention (i..e., Gray, 1961; Hess, 1962; Smith \& Hoyes, 1961) as has shape or form (i. e., Hess, 1962; Moltz, 1960; Ramsay, 1951). In addition, there is general agreement upon the major importance of auditory stimuli (i. e., Gottlieb, 1963; Ramsay; 1951).

Ramsay (1951) has reported that, in noting the differential effects of these different stimuli, auditory stimuli are more important than visual factors with form being less important than color. Therefore, in order to determine the differential influence of color, sound, and form on the early behavior of chicks with regard to preference and imprinting, the following experiment wàs undertaken.

\section{Subjects}

The Ss were 64 White Leghorn chicks obtained from a commercial hatchery and placed in laboratory incubators for the full developmental period. The eggs were placed in the incubators in groups of 20 every other day so that the hatch would be staggered in order to make it possible to test all chicks at 12-14 hr. of age. As soon as ringing began, the egg was placed in an individual compartment 6 in sq by 6 in deep with wire cloth floor and a solid lid except for a light and air aperture. Age of the chicks was recorded from the time of transfer. The Ss were removed only for the testing session. Neither food nor water was available in the isolation compartments.

\section{Apparatus}

Eight releasing combinations of form, color, and sound were used. The two forms were made of $1 / 4$ in plywood and were 6 by $51 / 2$ in: one was a rectangle, the other an outline of a hen. Each form was painted either red or white. The additional component of each combination was sound or no sound which was administered by a tape recording of a metronome set at 100 beats per minute.

The apparatus was a modified Gray-Pattie (1961) device. It consisted of a box painted the same gray as the isolation compartments, 38 in long by 12 in wide and 12 in high. The length was divided into three sections separated by glass. The center compartment floor, on which the $\mathrm{S}$ was placed, was a 13 by 12 in platform pivoted on the horizontal axis perpendicular to the longest dimension of the box. A weight of $20 \mathrm{gm}$, 2 in from either of the outer edges, would tilt the platform and activate microswitches beneath. The end compartments were alike except for the releasing combinations and were illuminated with $25 \mathrm{w}$ lights shaded from the $\mathrm{S}$. The box was covered by a lid to avoid any extraneous light or other stimuli.

The releasing objects were fitted in an upright position over an extension rod of the $1 \mathrm{rpm}$ motor shaft protruding through the floor of each end compartment. The lowest point of the object was about 1 in from the floor and the highest 7 in. The shaft was 6 in from the glass wall separting the end compartments from the inner one.

\section{Procedure}

The lights in the testing room were turned out and the chick was transported in his isolation compartment. The $\mathrm{S}$ was set in the apparatus with the long axis of its body perpendicular to the long axis of the box. If the chick wandered from the neutral position, it was recentered before the lights in the box were turned on. A test session was $15 \mathrm{~min}$.

-Each of the three factors of color, form, and sound was varied on two levels: red or white, rectangular or hen-shaped, and sound or no sound. The design was a $2 \times 2 \times 2$ factoral with an unreplicated latin square so as to test each one of the eight treatment combinations against all others plus itself. Each chick was employed for only one of the 64 possible comparisons.

Scoring was accomplished by assigning a plus one for each minute the clock registered the chick as preferring the treatment combination to which it belonged and a minus one for each minute the clock registered a choice for the combination being compared. For the time the chick stayed in the neutral position, the clock would not register. The difference between the two scores was taken as the score entered into statistical analysis.

It was assumed that without any objects present or with the same combination of factors in each end compartment, the mean value of a large number of sample scores would be near zero. 

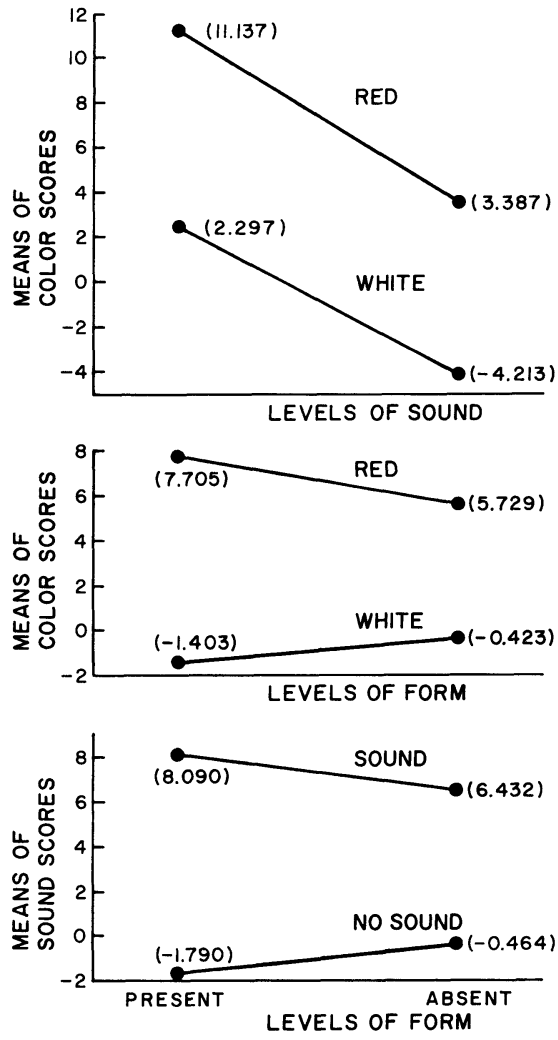

Fig. 1. Relations of sound, form, and color demonstrating the additive effect.

\section{Results}

The assumption just mentioned as well as any biasing of the apparatus itself was tested by noting the times when a treatment combination was paired with itself. Taking left-end compartment scores as compared to right-end compartment scores and using a two-tailed $t$ test, a nonsignificant value of .996 was found.

The sum of scores for each treatment was subjected to analysis of variance. The variance between treatments yielded an F of $3.404(p<.01)$. Bartlett's test for homogenity of variance was nonsignificant with $x^{2}$ of .008 . Sound yielded an F of $9.896(p<.01)$, color an $F$ of $12.890(p<.01)$, with form and all interactions being nonsignificant.

\section{Diseussion}

The nonsignificant $t$ found between the time spent in the left- and right-end compartment choices when both compartments held the same treatment condition demonstrated that the apparatus was not biasing the results and confirms the assumption of the mean value of scores being near zero if the end compartments contain the same factor combination.
The presence of either sound or color increased the preference for a treatment combination. This supports past research, though in this case color seems to be more important than sound (see Fig. 1). Form seems to have no effect.

The nonsignificant $F^{\prime} s$ dealing with the interaction between the stimuli would indicate that the action of color and sound are independent; that is to say, the effect of one does not differentially influence the effect of the other. However, the effect of sound, color, and form do seem to be additive, though form contributes little. As can be seen in Fig. 1, the combination of sound and red is much more preferred than a combination with one of them absent.

These results may be seen in light of an arousal hypothesis. It is generally assumed that a stimulus complex is associated with a certain arousal reaction and that there is a dimension of degree of such responses. Therefore, the intensification of the stimulus situation should be related to increased arousal. However, arousal is not to be seen simply as the total amount of stimulation impinging upon the organism (Pitz \& Ross, 1961), but rather the attention aspect of the stimulus complex. From this concept of arousal, it would be predicted that if imprinting is directly related to arousal the combination of stimulus factors would be more effective than either factor alone.

From this point of view, many of the observations made in regard to imprinting become coherent and understandable, for example, slight punishment or shock being beneficial, the massing of practice and expenditure of energy increasing imprinting effectiveness, and moving objects being more effective than stationary ones.

\section{References}

GOTTLIEB, G. Following-response initiations in duckling: age and sensory stimulation. Science, 1963, 140, 399-400.

GRAY, P. H. The releasers of imprinting: differential reaction to color as a function of maturation. J. comp. physiol. Psychol., 1961, 54, 597-601.

HESS, E. H. Ethology: an approach toward the complete analysis of behavior. In New directions in psychology, New York: Holt, Rinehart and Winston, 1962.

HINDE, R. A., THORPE, W. H., \& VINCE, M. A. The following response of young coots and moorhens. Behavior, 1956,9, 214-242.

MOLTZ, H. Imprinting: empirical basis and theoretical significance. Psychol. Bull., 1960, 57, 291-314.

PITZ, G. F., \& ROSS, R. B. Imprinting as a function of arousal. J. comp. physiol. Psychol., 1961, 54, 602-604.

RAMSAY, A. O. Familial recognition in domestic birds. Auk, 1951, $68,1-16$.

SMITH, F. V., \& HOYES, P. A. Properties of the visual stimuli for the approach response in the domestic chick. Anim. Behav., 1961, $9,159-166$.

\section{Notes}

1. A thesis submitted in partial fulfillment for Honors in Psychology at Whitman College by the senior author. For reprints, write the second author. 